\title{
Rezension:
}

\section{Zu Recht wieder Anwalt}

\author{
Zu Recht wieder Anwalt \\ Jüdische Rechtsanwälte aus Berlin nach 1945 \\ Hans Bergemann, Rechtsanwaltskammer Berlin (Hrsg.) \\ 2012 Hentrich \& Hentrich Verlag Berlin
}

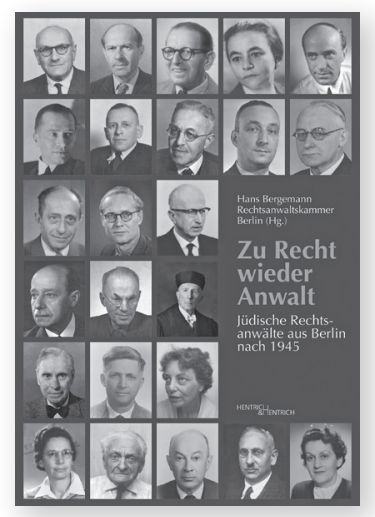

Nach den umfangreichen Recherchen, Veröffentlichungen und Ausstellungen der letzten Jahre unter dem Titel „Anwalt ohne Recht" nimmt sich die Rechtsanwaltskammer Berlin nun eines weiteren Themas der Geschichte der jüdischen Juristen und - damals - wenigen Juristinnen in Deutschland an. Wie ging es nach dem Ende des Zweiten Weltkriegs weiter, jedenfalls bei jenen, die die Shoa überlebt hatten? 2010 erteilte die Berliner Rechtsanwaltskammer einen entsprechenden Rechercheauftrag an den Historiker Hans Bergemann. Die Ergebnisse seiner Untersuchung sind nun unter dem Titel „Zu Recht wieder Anwalt" auf 308 Seiten beim Hentrich \& Hentrich Verlag veröffentlicht.

Das Buch enthält Informationen und biografische Angaben über

- überlebende deutsche Juristen (einschließlich jener, die vor ihrer Emigration noch in Ausbildung waren) jüdischer Herkunft, die sich nach 1945 als Anwälte (wieder) in Berlin niederließen,

- frühere Berliner Anwälte jüdischer Herkunft, die1945 nicht nach Berlin zurückkehrten, sondern sich in Westdeutschland niederließen,

- frühere Berliner Anwälte jüdischer Herkunft, die zwar nach Berlin zurückkehrten, aber sich nicht wieder anwaltlich betätigten,

- frühere Berliner Anwälte jüdischer Herkunft, die in verschiedenen Funktionen in Deutschland wirkten, ohne dass sie ihren Hauptwohnsitz dorthin verlegt hätten.

Die Untersuchung betrachtet außerdem exemplarisch die Wiedergutmachungs- und Entschädigungsverfahren für die verfolgten Anwälte. Die zahlenmäßige Zusammenfassung der Ergebnisse wird ergänzt durch ein biografisches Verzeichnis mit Kurzbiografien der ermittelten Personen und ein ausführliches Namens-, Quellen- und Literaturverzeichnis. Diese Angaben sind sehr hilfreich, erstens bei der Lektüre des Buches und außerdem für weitere Recherchen.

343 Juristen wurden anhand der genannten Kriterien ermittelt und außerdem sechs Juristinnen mit beeindruckenden Lebensläufen und auch beruflichen Erfolgen, deren Biografien stark verkürzt umfassen:
1. Anita Eisner (1900-1950) studierte von 1919 bis 1923 Jura in Berlin, 1927 zweites Staatsexamen, 1933 Berufsverbot, 1943 bis 1945 untergetaucht, Mutter und Schwester werden deportiert und ermordet, ab 1945 vorläufig wieder als Anwältin zugelassen, ab 1947 Notarin.

2. Dr. Hanna Katz (1895-1982) promovierte als erste Frau an der juristischen Fakultät der Berliner Universität, Gerichtsassessorin, ab 1930 Anwältin, 1938 Berufsverbot und Zulassung als „jüdische Konsultentin“, 1941 Emigration in die USA und erneutes Jurastudium mit bar exam 1946, 1954 Zulassung als Anwältin in Berlin ohne Residenzpflicht.

3. Dr. Edith Krojanker (geb. Epstein, 1905-1995) studierte 1924-1927 Jura in Königsberg und Berlin, 1929 Promotion an der Universität Königsberg, 1932 zweites Staatsexamen, Gerichtsassessorin an Berliner Gerichten, 1933 Berufsverbot und Emigration nach England, Palästina, 1948 Anwaltsprüfung in Israel, 1949 Zulassung zur Anwaltschaft in Israel, ab 1953 Tätigkeiten in Deutschland für die Jewish Trust Corporation und als Rechtsanwältin im Regierungsdienst.

4. Dr. Käthe Loewy (später: Manasse, 1905-1994) studierte Jura in Freiburg, Berlin und Bonn, 1931 Promotion an der Universität Bonn, 1932 zweites Staatsexamen, Gerichtassessorin in Berlin-Schöneberg, 1933 Zulassung als Rechtsanwältin und drei Monate später Berufsverbot, 1938 Emigration nach Palästina, 1942 Deportation und Ermordung der Mutter, 1949 Rückkehr nach Deutschland, 1953 Richterin am Landgericht Hamburg, später Landgerichtsdirektorin.

5. Hilde Neumann (geb. Rosenfeld, gesch. Kirchbeimer, 1905-1959) studierte Jura in Berlin, Freiburg und Bonn, 1925 Eintritt in die SPD (wie ihr Vater Kurt Rosenfeld), nach zweitem Staatsexamen 1932 Rechtsanwältin am Kammergericht, 1933 Berufsverbot, bis 1939 Mitarbeiterin der Internationalen Roten Hilfe, 1936 nach einjährigem Studium des sowjetischen Justizwesens Eintritt in die KPD, 1940 nach Lageraufenthalt Emigration nach Mexiko, 1947 Rückkehr nach Deutschland, Eintritt in die SED, verschiedene Aufgaben beim Aufbau des Justizwesens in der EBZ und DDR, 1949-1950 Präsidentin des Landgerichts Ost-Berlin, dann Magistratsdirektorin für Justiz in Berlin, 1953-1959 Chefredakteurin der „Neue Justiz“.

6. Erna Proskauer (geb. Aronsohn, 1903-2001) studiert Jura in Berlin und Freiburg, 1932 zweites Staatsexamen, 1933 Berufsverbot und Ablehnung des Antrags auf Zulassung als Rechtsanwältin, Emigration nach Frankreich und 1934 nach Palästina, 1951 Antrag auf Wiedereinstellung in den Justizdienst (Ablehnung 1956), 1953 Rückkehr nach Berlin, 1957 Antrag auf und Zulassung zur Anwaltschaft, ab 1962 Notarin.

Was bedeutet diese Zahl „349“? Mitte 1933 hatte das Deutsche Reich mehr als 65 Millionen Einwohner, darunter etwa eine halbe Million Juden. Etwa 5.000 der um die 19.200 im Deutschen Reich 1933 zugelassenen Rechtsanwältinnen 
und Rechtsanwälte wurden von den Nationalsozialisten als „nichtarisch“ definiert. Ein Drittel der deutschen Juden wohnte in Berlin. Daher gab es dort auch die meisten jüdischen Rechtsanwälte und Rechtsanwältinnen. Es waren am 1. Januar 1933 etwa 1.800 Anwältinnen und Anwälte von insgesamt 3.400 Berliner Anwältinnen und Anwälten.

Etwa 400.000 Deutschen jüdischer Herkunft gelang es, das Deutsche Reich rechtzeitig zu verlassen und am Leben zu bleiben. Ungefähr 15.000 deutsche Juden überlebten die Konzentrationslager, im Untergrund oder als Ehepartner/in von Nichtjuden. Nach 1945 kehrte nur eine vergleichsweise geringe Zahl aus der Emigration nach Deutschland zurück. Die meisten zogen es vor, Deutschland fern zu bleiben. Bis 1989 lebten nicht mehr als 30.000 Juden in Deutschland. Da Juristinnen und Juristen jedoch größere Schwierigkeiten haben, ihren Beruf in einem anderen Land mit fremder Sprache auszuüben als etwa Ingenieur/inn/e/n oder Ärzt/inn/e/n, dürfte auch der Rückkehreranteil unter den Juristen entsprechend höher sein. Hierzu ein
Zitat von Erna Proskauer, das auch Hans Bergemann wiedergibt: „Gerade Juristen mit ihrem örtlich begrenzten beruflichen Anwendungsgebiet fanden nirgends im Exil, vor allem aber nicht in den engen Grenzen der israelischen Wirtschaft, eine Erwerbsmöglichkeit, geschweige denn eine angemessene.“ 349 ist nicht viel. Zumal sich von 1945 bis 1990 nur 179 Anwält/inn/ e/n jüdischer Herkunft zeitweise oder dauerhaft (wieder) in Berlin niederließen, die 1933 bereits als Anwält/inn/e/n oder Beamt/ inn/e/n im höheren Justizdienst tätig gewesen waren oder sich 1933 in ihrer juristischen Ausbildung befunden hatten. Und auch das Kapitel, das über die Grundlagen der Rückerstattung und Entschädigung für die verfolgten jüdischen Rechtsanwältinnen und Rechtsanwälte berichtet, ist deprimierend.

Die Rechtsanwaltskammer Berlin hofft, mit diesem Band dazu beizutragen, dass das Schicksal der jüdischen Kolleginnen und Kollegen nicht vergessen wird und auch künftigen Generationen zugänglich bleibt. Dieser Hoffung schließe ich mich an. Anke Gimbal, Berlin

\section{Rezension:}

\section{Rechtsnormen des StGB suchen, verstehen und auslegen}

\section{Otto Lagodny}

Rechtsnormen des StGB suchen, verstehen und auslegen

Eine Anleitung für die ersten Schritte im Strafrecht

2012, Buch, $203 \mathrm{~S}$.

nwVerlag IDBN 978-3-7083-0860-9

Wien, Graz 2012

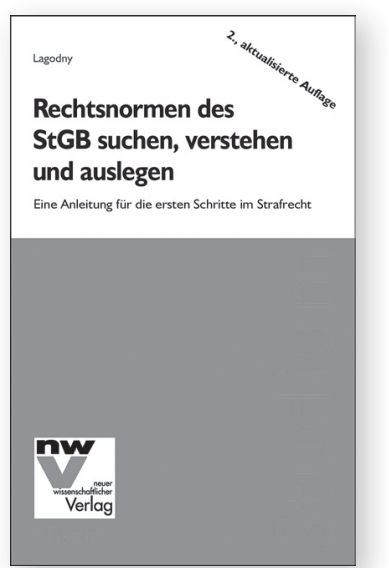

Bei dem Buch des Salzburger Professors Otto Lagodny handelt es sich um ein Lehrbuch für Studierende der Rechtswissenschaft in den ersten Semestern. Es muss allerdings zu Beginn angemerkt werden, dass das Buch für österreichische Studierende verfasst wurde und vor allem für diese nützlich ist, da es sich auf österreichische Rechtsnormen bezieht

Das Buch ist in mehrere Abschnitte unterteilt: Nach einer Einführung gibt es ein Kapitel über das Finden einer Norm im StGB, eines über das Lesen und Verstehen der gefundenen Norm, ein weiteres über das strafrechtsbezogene Lesen und ein abschließendes kurzes Kapitel, das sich mit dem schnellen Wieder-Erkennen der Normen befasst.

In dem Kapitel über das Finden einer Norm stellt der Autor zunächst den Aufbau des Strafgesetzbuches dar und erläutert kurz den Inhalt der einzelnen Abschnitte. Er gibt zahlreiche „Suchbeispiele“, wie er es nennt, dafür, wie man eine be- stimmte Norm auffinden kann, all dies anhand kleiner Fälle, deren Lösung am Ende präsentiert wird.

Der Abschnitt über das Lesen und Verstehen der gefundenen Norm befasst sich in erster Linie mit den Auslegungsmethoden von Rechtsnormen. Diese werden anhand einer größeren Auswahl von Strafrechtsnormen des StGB erläutert und mit Beispielen unterlegt.

Das Kapitel über strafrechtsbezogenes Lesen stellt die Verbindung zwischen Allgemeinem und Besonderem Teil des StGB her, wobei der Allgemeine Teil als „Werkzeugkasten“ für den Besonderen Teil bezeichnet wird. Auch hier wird umfangreich auf Normen und Beispiele Bezug genommen. Dabei wird in gebotener Kürze der Allgemeine Teil abgehandelt, so dass den Studierenden ein erster Eindruck über diesen vermittelt wird

Im letzten Abschnitt, der das Wieder-Erkennen von Normen zum Inhalt hat, geht es in erster Linie um die Anwendung des in den vorherigen Kapiteln gelernten im Rahmen einer Klausur. Hier soll vermittelt werden, wie die Studierenden auch unter Zeitdruck die Normen schnell und effizient wiederfinden und anwenden können. Auch die Vorbereitung der Klausur durch Unterstreichung der wesentlichen Bestandteile einer Rechtsnorm wird dabei abgehandelt.

Insgesamt ist das Buch für Studienanfänger/innen sicherlich empfehlenswert, weil eine sichere Beherrschung der Methodik eine unerlässliche Grundlage für ein erfolgreiches Studium der Rechtswissenschaften ist und diese hier umfassend dargestellt wird. Die Besonderheit dabei ist, dass die Methodik hier ganz speziell auf das Strafrecht abgestimmt wurde. Ein wenig negativ fällt dabei allerdings auf, dass in dem Werk bereits im- 